\title{
The Impact of Multidisciplinary Teams on Sustainability Projects in EPICS
}

\section{Dr. Stephanie M. Gillespie, Arizona State University}

Stephanie Gillespie joined the EPICS@ ASU program after finishing her Ph.D. in Electrical and Computer Engineering from the Georgia Institute of Technology. She has extensive experience in K-12 outreach and curriculum development, and is passionate about giving students opportunities to make a difference throughout their academic career. As the EPICS Director of Instruction, Stephanie leads the EPICS program's curriculum development, EPICS-Community College program, and program assessment efforts. She received her MSECE from Georgia Tech and her BSEE from the University of Miami.

\section{Mr. Mark Vincent Huerta, Arizona State University, Polytechnic campus}

Mark Huerta is a $\mathrm{PhD}$ candidate in the Engineering Education Systems \& Design program at Arizona State University (ASU). He earned his BS and MS in Biomedical Engineering, both from ASU. He is the Co-Founder \& Chairman of 33 Buckets, a non-profit that provides sustainable clean water access in the developing world. Mark has experiences as a researcher, social entrepreneur, engineer, teacher, and higher education program manager.

Mark's research interests revolve around developing engineers capable of leading and enacting positive change on their communities. His research explores the topics of entrepreneurial mindset, innovation, well-being, leadership, interpersonal skills, and other 21st century competencies. Mark has experiences in teaching and mentoring engineering students in human-centered design, social entrepreneurship, humanitarian engineering, leadership, and mindfulness.

\section{Dr. Jared Joseph Schoepf, Arizona State University}

Jared Schoepf is the Director of Operations for Engineering Projects in Community Service (EPICS) at Arizona State University. Jared received his $\mathrm{PhD}$ in Chemical Engineering at ASU, developing a tiered approach to rapidly detect nanomaterials in the environment and consumer products. Jared has been a lecturer of EPICS for 4 years, mentoring over 200 teams. Currently he teaches introduction to engineering, EPICS, and chemical engineering courses. He has founded 2 starts ups and has 3 patents for water purification, removal of trash from storm water, and antibacterial liquid hand soap formula. He has a passion for teaching and mentoring students, aiming to help each student achieve their goals

\section{Mr. Joshua Loughman, Arizona State University}

Joshua Loughman is a Lecturer for the Ira A. Fulton Schools of Engineering at Arizona State University. $\mathrm{He}$ is the Director of Development for the Engineering Projects in Community Service (EPICS) program. His research interests are in engineering education, sustainable engineering, and science and technology studies. 


\title{
The Impact of Multidisciplinary Teams on Sustainability Projects in EPICS
}

\begin{abstract}
The Engineering Projects in Community Service (EPICS) program is a service-learning program founded by Purdue University in 1995 and is now an active part of the Arizona State University curriculum. Compared to traditional service learning, EPICS differs by implementing student learning through design projects with real community partners, multidisciplinary teams, industry mentors, and projects that span multiple semesters with potentially new student teams every semester. In this work, the multidisciplinary team factor is examined due to the potential benefits and challenges of supporting multidiscipline teams in an academic curriculum. While multidisciplinary project-based learning and multidisciplinary service-learning are not new ideas, rarely is the team composition considered in relation to the impacts to student learning and perception.
\end{abstract}

This work examines the experiences of three multidisciplinary, sustainability focused teams providing solutions for use and education in communities considered food-deserts. The three team structures vary in degree of multidisciplinary composition, one of the EPICS differentiators. Students were asked to define multidisciplinary teams and then reflect on their own team experiences and team compositions. Transcripts of focus group interviews with current and previous student team members were analyzed to determine the extent to which multidisciplinary composition of the student teams impacted student perceptions of project success, skills acquired, and overall team environment.

To complement the student perspectives, faculty perspectives regarding supporting multidisciplinary teams in the EPICS program were also collected through a roundtable discussion. Results of a roundtable and SWOT (Strengths, Weakness, Opportunities, Threats) analysis are included and discussed. This paper reports the results of the student-focused and faculty-focused analysis of multidisciplinary EPICS teams and plans for further work.

\section{Introduction}

The Engineering Projects in Community Service Program was started at Purdue University in 1995 as a means of providing students with academic credit through participation in long-term service-learning projects. Student teams are vertically-integrated multidisciplinary teams, comprised of students of all years of undergraduate study and multiple majors [1]. Student outcomes are often summarized to include technical skills, communication skills, organizational skills, teamwork experiences, resourcefulness, resource management, sponsor awareness through customer and client interaction, expanded community awareness, and professional ethics [1]. Over 30 institutions across the US have an EPICS program, as well as multiple institutions abroad. The EPICS program was implemented at Arizona State University in 2009 and has since grown to approximately 200 students participating every semester in a one-credit class that may or may not contribute to degree requirements depending upon the students' majors. 
One key aspect of the EPICS program at ASU is the multidisciplinary aspect of the team. A multidisciplinary team can contribute to the 2019-2020 ABET Criteria 3 and 5 which specify

and

$$
\text { "an ability to communicate effectively with a range of audiences" }
$$

$$
\begin{aligned}
& \text { "an ability to function effectively on a team whose members together provide } \\
& \text { leadership, create a collaborative and inclusive environment, establish goals, } \\
& \text { plan tasks, and meet objectives" [2]. }
\end{aligned}
$$

Prior versions of the ABET criteria included the Criteria 3.d, "an ability to function on multidisciplinary teams," but this was last seen in the 2017-2018 Criteria [3]. However, there are very few opportunities for students to have multidisciplinary experiences in their coursework. Most undergraduate students experience multidisciplinary teams in only a small subset of their coursework, including introduction to engineering classes taken by first-year students. The traditional engineering program is most often taught from the basis of the selected discipline of the degree program with little opportunity for breadth [4]. Due to the challenge assessing a student's ability to meet ABET Criteria 3.d [5], many capstone courses are conducted as school-specific offerings to ensure appropriate closely supervise the curriculum and assess learning outcomes to ensure program accreditation. The EPICS Program is in a unique position to offer multidisciplinary experience to engineering students because it is focused on servicelearning rather than discipline-based knowledge. At ASU, the program is directed through a group of faculty under the supervision of the Academic and Student Affairs unit, which also works with student engagement, first year engineering, the Grand Challenge Scholars Program, and other similar programs. Anecdotally, the teams that were most successful in the ASU EPICS program were those that incorporated a diverse group of students from multiple majors. This observation was the motivation for this work.

The study of teamwork, especially multidisciplinary teams, is not a new area of interest. Borrego and Newswander identified 5 themes of assessing multidisciplinary work: disciplinary grounding, integration, teamwork, communication and translation across discipline boundaries, and critical awareness [6]. Multiple studies have explored the aspects of multidisciplinary team outcomes for capstone design teams $[7,8]$. Other studies have explored the possibility of multidisciplinary teams in a variety of other courses including having a multidisciplinary group of faculty teach general engineering classes during the first-two years of study [4].

Since service-learning projects often require both engineering and non-engineering knowledge for successful deployment, having students from a variety of backgrounds, including nonengineering, can be valuable. While engineering service-learning is poised as an excellent platform to promote multidisciplinary teams, there are many challenges that accompany it. Very few studies have explored multidisciplinary engineering teams in service learning. Previous research from the EPICS program identified some of the challenges with multidisciplinary teams in a service-learning environment, and proposed solutions of multidisciplinary faculty and teaching teams, generalized learning outcomes, and engineering tools that transcend disciplines [9]. Other research work on the EPICS program investigated team social responsibility [7]. Most 
recently, the Frontiers in Education 2018 conference hosted an entire track devoted to EPICS and service learning [10]. In this paper, we add to the body of knowledge on multidisciplinary teams in service-learning environment by identifying student perceptions of working in multidisciplinary teams, faculty perceptions of facilitating multidisciplinary teams, and institutional support for multidisciplinary teams.

\subsection{EPICS at ASU}

In Fall 2009, Arizona State University adopted the EPICS program with a 1 credit class offering with an initial enrollment of 35 students eager to work on a real project with a real impact. Students ranked their top three project ideas with the professor forming teams based on interest. Teams were primarily composed of engineering students from all engineering degree programs, freshman to seniors. Throughout the last ten years, the projects focused on four themes: sustainability, education, community development, and health. While the themes remained the same, the method of team formation, the goals of the teams, and the mentorship have changed with a shift in direction by the program leadership. From 2009 until 2013, teams were paired with a community partner, a faculty member, and an industry partner to aid in the design, build and delivery of the project. Teams used the human-centered design process to identify the problem and design a solution, with a focus on social entrepreneurship. Teams had no funding and were required to apply for grants locally at ASU, nationally, and globally bringing awards and recognition to the EPICS at ASU program. With the change in program leadership in 2013, the focus on social entrepreneurship shifted to a focus on meeting the needs of the community partner.

Under new leadership, teams focused on the needs and requirements of the assigned community partner, creating individualized solutions with the goal of increased delivery of solutions. The previous focus on entrepreneurship increased the time for project delivery as students shifted class time to developing a business, marketing, and sales plans. A deeper connection with community partners and their respective needs were a main result of the individualized design solutions. While the focus shifted from social entrepreneurship to community partner individualized solutions, teams continued to impact their community. Teams who successfully deliver a project were then encouraged to investigate the social entrepreneurship pathway.

Over the past ten years, through the application of grant funding, teams have been awarded in excess of $\$ 500,000$. Projects have delivered a mobile application to connect leftover food from events to food banks, a water filtration system in Bangladesh, and a maternity clinic in Kenya. Students have developed and applied skills in needs assessments, teamwork, problem solving, pitching, and building.

Team formation in the EPICS at ASU Program has evolved over the years. In the current format, teams are formed by balancing the preferences of the students, the project needs, and the availability of mentors in order to achieve the twin goals of project success and student learning. Each team is organized into a group of usually 4-10 students that are available during one of the several "team meeting" time slots and various criteria are weighed such as type of desired project, seniority (both year in college and number of semesters within the program are considered), major, and other experience or skills that may be relevant to project success. A 
matching process is used to assign teams mentors who are local professionals or other subject matter experts (sometimes graduate students or university staff are used as mentors). The composition of teams is balanced to achieve both the total team size and team diversity thought to bring about two goals: making progress on the project and providing the best opportunity for students to learn from their project, their teammates, and their community partners.

One of the key differentiators the EPICS program has from other project-based learning or team design courses is that projects can persist for multiple semesters. This provides an interesting student and program challenge to provide for the management of these projects. Experiences with on-boarding, transitions, client communication and expectations setting become important skills for the teams to develop. Since the program operates in a one-credit per-semester course, students are free to continue with their current project, elect to join a new project, or leave the program entirely. This means that nearly every project will have a different composition of students as the project evolves. Sometimes this is necessary because of student selection and sometimes it is based on the changing needs of projects at different points in their life-cycles. For example, experience with fabrication and manufacturing engineering may be needed in abundance toward the end of a project and so special care might be taken to recruit such students to join at that phase of a mature project team.

\section{Background on teams}

While the terms interdisciplinary and multidisciplinary traditionally differed in definitions, in recent years academics and practitioners have used them interchangeably [6]. While no specific definition of multidisciplinary is used throughout this work to avoid applying preconceived notions to the review of student and faculty perceptions, a general accepted definition of multidisciplinary implies the inclusion of at least two distinct backgrounds or approaches [6]. The advantages of diverse teams has become an accepted outcome from both academic and industry perspectives. Hong and Page developed a model for understanding the inherent advantages of diverse teams even when compared to homogenous groups of superior problemsolvers [11]. In their model, a diversity of backgrounds, training, experience, and identities lead to a group with more diverse problem-solving heuristics from which to draw from when confronted with a novel problem.

As one example, researchers have investigated the benefits of a multidisciplinary, senior engineering capstone course of mechanical engineering (ME) and biomedical engineering (BME) students [8]. Combining the disciplinary expertise of both majors was theorized to create more valuable final products as BME's provided regulation expertise and ME's provided mechanical design expertise. Although the study did not investigate student perspective of the course, the study found "that students in multidisciplinary capstone courses have, on average, a higher likelihood of being employed seven months after graduation."

Teamwork has been a consistent theme throughout the study of student learning outcomes from EPICS service learning [1], with approximately $85 \%$ of students in a prior study of EPICS students denoting teamwork as one of the most valuable things they learned from being a part of EPICS [12]. Student perspective on project success has been difficult to characterize on a class by class or year by year basis due to challenges in defining success. In one study of business 
students, the "five key student themes identified in support of team-based learning included the following: (a) better deliverables, (b) increased ideas, (c) improved learning experience, (d) reduced workload, and (e) collective security" [13]. In conclusion the study found teams allowed students to build upon each other's ideas with a final solution which would not have been produced solely by a single student. The study also found that students who prefer working alone stated their reasons including, "(a) grade reciprocity, (b) social loafing, (c) schedule challenges, and (d) work and school team differentiation." Practitioners have identified key areas to address the negative aspects of teamwork through the implementation of team norms or team agreements, peer-evaluation of team members, and team timelines to improve work efficiency and clear objectives to be successful $[14,15]$.

\subsection{Student perceptions of teams}

Work by Burian et al. highlights an example project based learning for teaching sustainability, but included only nominal comments by the students on the impact of the multidisciplinary groups they worked in, spanning from "I focused on my design and did not interact very much with the other students" to "Collaborating with other student groups helped to expand the scope of work to include ideas that were novel and interesting" [16]. According to Rabb's study, students working on the multidisciplinary based team expressed an increased agreement with a variety of statements including "My motivation to learn and continue learning has increased" and "My ability to identify and formulate engineering problems and apply my knowledge of math, science, and engineering along with creativity skills improved," compared to general capstone experiences within single-discipline project teams [17].

Miller and Olds found in an entry-survey of a multidisciplinary senior design class that $80 \%$ of students agreed that multidisciplinary engineering teams tend to produce better engineering design, and this percentage did not change much over the two-course sequence [18]. They also found that students in the multidisciplinary senior design were more likely to grow to appreciate engineering synthesis in their design work by the end of their course experience, a trend not seen in students in single-discipline capstone experiences.

\section{Methods}

In this study, three different aspects of data were collected to encompass the full scope of student learning outcomes through multidisciplinary teams in service-learning programs such as EPICS. The first data set was collected through ASU-EPICS student focus groups and interviews regarding their perception of their multidisciplinary team experience. The second data set was collected at the 2018 Global EPICS Symposium and Workshop through both a guided faculty/staff conversation as well as creation of SWOT artifacts by attendees. The last set of data was focused on programmatic inclusion/exclusion of and support of multidisciplinary teams and was collected via a targeted distributed survey to program directors or lead faculty at EPICS Programs across global institutions. IRB approval was obtained by Arizona State University for the studies. 


\subsection{Data collection:}

To study the student perceptions to multidisciplinary teams in the EPICS program, a focus group interview protocol was developed that attempted to elicit student feelings and perceptions of the team experience. ASU students were first asked about their general EPICS experience and why they joined their project, and then about their experiences on their specific team. The second stage of the interview asked students to define project success in the EPICS program. The third stage of the interview asked students to define multidisciplinary, considering what number of engineering and/or non-engineering majors needed to be included to have a team be multidisciplinary. Students were then asked to reflect on if their multidisciplinary experience had any specific outcomes, benefits, or inhibitors, and to redefine project success. While the intention was to collect the data through multiple focus groups, many of the focus groups turned into individual interviews due to student schedules and the timeline of the project. In total, there was one focus group and four additional individual interviews. Although the goal was to have focus groups, the individual interview processes possibly resulted in minimizing confirmation bias and tangents taken by the students.

While student perceptions were viewed as important, faculty/staff perceptions of supporting multidisciplinary teams were considered equally important since there are often many additional complications that can arise when including multidisciplinary teams in a classroom environment. An additional focus-group interview protocol was developed that complemented the student protocol. Participants were asked to describe the main goals of their EPICS program, including key student learning outcomes and skills taught by the faculty. This was followed by a conversation to attempt to define what types of students are required to call a team multidisciplinary, and concluded with programmatic support or barriers to supporting multidisciplinary teams. Participants were then asked to work in small groups and instructed on how to create a SWOT (strengths, weaknesses, opportunities, and threats) analysis of their programs focused on program-level support of multidisciplinary teams. Groups had ten minutes to complete the SWOT analysis.

Based off of the conversations from the faculty focus group, the importance of institutional support became a key component to address. A program-level data collection survey was created that asked for program-level goals, student and team demographics regarding student majors, and a question to understand which students at each institution could earn academic credit towards their degree through the EPICS program.

\subsection{Recruitment}

Specific teams invited to participate in the focus groups were selected based on the alignment of the project theme to the category of "Sustainability" and to the sub-theme of "Food Systems". Additionally, care was taken to select teams that had a spectrum of engineering and nonengineering majors present on the team. Since the composition of the teams is fluid over various semesters the overall contribution of multidisciplinary student members was considered rather than simply the most recent snapshot of student membership. Invitation emails were sent to approximately 25 students comprising from 5 different teams. Students were informed pizza would be provided during the focus groups. 
The faculty focus group was submitted and selected as a session as a part of the 2018 Global EPICS Symposium and Workshop. All participants at the symposium were informed of the session and invited to attend if they chose. Other sessions were scheduled concurrently so all participants opted-in to the session and were informed at the beginning that the session was a part of a research study, with consent forms required for all attendees. There was no incentive provided for participation in the faculty focus group.

To collect the institutional EPICS program-level data, individual contacts for 21 colleges or universities with active EPICS programs were invited by email to fill out an online Qualtrics survey. Participants were informed in advance that they may need to prepare some data analysis on their student teams in order to fill out the team demographic aspects of the survey. Participants were informed that those who successfully completed the survey would be able to receive a financial stipend provided by the Kern Foundation.

\subsection{Demographics}

A total of 7 students participated in the student data collection from the approximately 25 invited. The students who participated were juniors and seniors from 7 different engineering majors and all had at least two semesters of EPICS experiences on various teams. Two of the seven students were female. Additionally, the students identified as a broad range of ethnicities and races. Due to the small nature of some of the EPICS teams, their specific demographic information will remain unpublished to ensure anonymity.

Twelve faculty or program staff from various institutions chose to participate in the workshop faculty round table led by two faculty from ASU. The participants represented both domestic and international universities, as well as a variety of Carnegie classification institutions. Two of the twelve participants were female and the others identified as male.

Of the 21 colleges or universities invited to submit program-level data regarding teams, nine schools participated. Five of the nine respondents were female. Respondents held the title of Program Directors, Faculty Instructors, Lecturers, Managers, and Team Advisors. Types of institutions represented included a community college, state universities, and private universities.

\section{Data analysis:}

The student focus group and interviews were audio recorded, transcribed, and uploaded to Dedoose, a qualitative analysis software [19]. A thematic analysis was used to identify major patterns within the narrative. The best practices for completing a thematic analysis recommended by Braun and Clarke [20] and Creswell [21] were adhered to. The researcher that facilitated the interviews and focus groups initially made several passes through the data and then generated an initial codebook consisting of six themes and twenty-four codes. An inter-rater reliability test was completed, which involved a second researcher applying this codebook to the data. A pooled Cohen's kappa score of 0.44 was produced suggested poor to fair agreement $[19,22,23]$. The two researchers discussed the codebook and agreed that the discrepancies in the coding application test were mainly due to redundancies and overlap in several of the codes. A new 
iteration of the codebook was generated consisting of six themes and fourteen codes. Once again, a code application inter-rater reliability test was completed. This time the pooled Cohen's kappa score was 0.73 indicating good agreement $[19,22,23]$. The agreed upon final codebook was then used to write the results section.

The themes from the student focus group were similarly coded in the faculty round-table discussion transcriptions. However, other program-level codes were developed that were unique to the faculty and staff program perspective. Both student-codes and program-level codes were similarly coded into the SWOT analysis that the participants created.

Program-level survey data was analyzed for common themes and a comparison of priorities, statistics, and degree-requirement course applicability. Due to the small number of participants, the analysis of the program types is intended to complement the interviews and should not be considered conclusive on its own.

\section{Results}

\subsection{Student interview results}

In the student interviews, a total of six themes were identified: motivation for participation, program-level factors, skills learned, respect for other disciplines, team dynamics, and when is a project is successful. Each theme had between two and three individual codes identified with it. Table 1 shows the code book used in this work. While the themes somewhat follow the structure of the interview protocol, many aspects of the interview protocol were not emphasized by students to be critical to their experiences in EPICS.

Table $1-$ Final code book from student interviews

\begin{tabular}{|l|l|}
\hline Theme & Codes \\
\hline Motivation for Participating & Make a difference, Gain real world experience earlier \\
\hline Program-Level Factors & Project phase, Community partner interactions \\
\hline Skills Learned & $\begin{array}{l}\text { Engineering design process, technical skills development, } \\
\text { improved communication }\end{array}$ \\
\hline Respect for Other Disciplines & $\begin{array}{l}\text { Non-engineering perspectives, Recognizing other perspectives } \\
\text { (generic), Recognizing disciplinary expertise in engineering }\end{array}$ \\
\hline Team Dynamics & Team motivation, Individual student interests \\
\hline When is a Project Successful? & Community Partner Satisfaction, Creating Deliverables \\
\hline
\end{tabular}

The first student theme was when students highlighted their motivation for participation in the EPICS program; the most common responses were to make a difference and gain real-world experience earlier. Students emphasized that the EPICS program provided them with an opportunity to practice engineering before many of the design courses within their major. One 
student commented on their desire to "solve an actual issue without actually having to go through so many theoretical classes at the upper level and having to wait years to actually make a difference." Most students did not care if the EPICS project was within the scope of their own major or not, and some explicitly chose projects outside of their field of study to spend time learning topics outside of their assigned coursework.

These conversations led into the second theme of students discussing the theme of program-level factors, such as the current stage of a project (i.e., the start of a project, near the end) or the level and/or scope of community partner interactions. Program level factors were often discussed as having either a positive or negative impact on the student depending on the specific situations. Some students identified them as a reason for joining a specific project, i.e., "They were already testing out certain things. It was no longer an idea. It was actually making the idea into reality piqued my interest." Others mentioned the importance of the community partner for continuing on with a project for multiple semesters: "I feel like the project has to have some actual traction or kind of force behind it. I've seen a lot of projects where the community partner just kind of throws something out there and expects it not to happen and doesn't really care if it does." None of the students attributed their reason for joining or remaining on a project due to the team-nature of the projects or the opportunity to work on a team of any specific type.

The next phase of the student interviews asked students to define success for an EPICS project. Students usually identified project success as achieving community partner satisfaction with the scope of work thus far or delivery of a solution. This was exemplified by student comments including: "I would define project success as meeting the community partner's needs" and "Yet it was still a successful semester because our community partner was satisfied with our work to get that much closer to the goal." This viewpoint emphasizes that students don't feel the need to deliver a solution every semester to feel successful as an EPICS team as long as their community partner was happy and/or they were making progress towards a solution. Students were then explicitly asked what the majors of their prior team members were (if they remembered) and again asked to identify project success. Only one interviewee introduced the concept of teammember development and team dynamics as an aspect of EPICS team success and project success. The others continued to focus on community partner satisfaction and project delivery.

The interviews then shifted focus to general team interactions. Students formalized the importance of the team dynamics theme, including both positive and negative aspects of working in a team, often as it related to team motivation. Coded excerpts included: "My first semester within EPICS was in a group that had absolutely no direction. I had lost so much faith in what undergraduates can do," and "Once again it comes down to motivation. If people don't want to be there, they're not going to be there and not show up. So you have to give them a reason to come and whether that's by giving them more responsibility or less responsibility, just to make sure that they come really enhances." Additional team dynamics were discussed by observing that student interests and student majors do not necessarily align. Many students commented on individual members of their team that were critical to their team because of a passion or interest they had outside of their degree program. Two student examples include "Our specific project involves horticulture and everything, so I knew nothing about growing plants before, but somebody else on our team, they have a garden in their backyard, so they're a little bit more knowledgeable, so it's nice to gain some knowledge about that," and "I have to agree. I think 
some people ... I think some based on their skill sets are very interested. Some people's skill set interests relate to their major and some don't."

Students also introduced an additional theme of specific skills learned through their EPICS projects when elaborating about their previous teams. Many students mentioned that communication skills, including leadership and team-management, were a critical takeaway of the EPICS program. Often communication examples were directly linked to working with members of different backgrounds:

"I originally think a big skill I've gotten through EPICS is learning how to relate, how to take on my project and make a presentation about it so that people can understand it. Learning exactly how in depth with the scientific material I should get and more realistically how I should make it approachable because we do a lot of presentations, and so I think generally presentation skills is something I've been briefed through EPICS."

As another perspective, one student mentioned, "actually learned how the BME guys talked. I learned how the chemical guys talked, and so by getting that extra exposure, I'm actually able to, in the workplace, where I'm working right now, I'm able to understand where they are coming from." Other skill development focused on an understanding of the human centered design process and technical skill development.

In order to understand student perceptions of multidisciplinary teams, students were asked to define multidisciplinary teams, and then how many students of different backgrounds would need to be included. Many students agreed that a multidisciplinary team needed at least two students of various backgrounds, but few were unwilling to commit to a specific minimum number of majors or students. When asked to describe engineering multidisciplinary teams, students often defaulted to saying the various backgrounds could just come from one of the various engineering disciplines. Students were then asked if non-engineering students were required to be a part of a multidisciplinary team. Approximately half of the students thought it was necessary, while the other half did not think it was. However, almost all of the students saw the value-added by non-engineering students. One student responded, "I think that you can still be an interdisciplinary team with just engineers of different focuses, but I would once again say that you benefit from having a non-engineer." Another mentioned, "I don't think they need to, but I think it definitely means more to be multi-disciplinary because engineering is a discipline on its own, whether you're mechanical or whatever. Business is a discipline so I feel like it's both, you can have a multi-disciplinary engineering group but if it's just multidisciplinary then I don't really know."

The conversations relating to multidisciplinary teams led to the final theme seen throughout all of the student responses: respect for other disciplines. All seven of the students interviewed incorporated some direct comment regarding the value that other disciplines can add to a EPICS team. While some students focused more on recognizing other perspectives from a broad viewpoint (not focusing on student majors), others focused on the importance of including various types of engineering majors and others focused on how non-engineers add value to an 
EPICS team. Some of the student perspectives highlighting a respect for other disciplines include:

"Having a member with seniority that was very well versed in entrepreneurship in small product development aided a lot in providing us a centralized point of view in how we should approach with the community partner."

"I think it was diversity of thought. Having different people ... when different people approach the same problem in different ways, I think you form better solutions and that's I think I enjoyed most."

"I just have to say we need to get more people from different majors."

"But if you're electrical, obviously you have more knowledge in circuits than an aerospace engineer will. Or an aerospace will have more experience in thermo or dynamics management than a BME will. So everyone can still offer knowledge."

\subsection{Results of faculty focus group}

The six themes were also used to code the transcript of the faculty focus group and SWOT analysis. Student motivations and program factors were not mentioned by the faculty members since the focus group protocol did not focus on these questions due to time constraints. The faculty thoughts on skill development echoed that of the students: focus on human centered design, communication, and technical skill development. However, the faculty focus group also suggested many broader learning outcomes or skills learned that were distinct from those of the students: problem solving capacity, critical thinking, project management, civic engagement, and handling community partner interactions.

When defining EPICS project success, the faculty members emphasized that it was more important that the students are making progress in the design process rather than either making the community partner happy or creating deliverables. While both of those were viewed as ideal targets, they were not necessary to define a successful EPICS project. Some of the faculty expressed that mastering team dynamics (including team motivation and incorporating individual student interests) were more important than deliverables or community partner satisfaction.

The faculty group had a lot of conflicting ideas regarding the definition of multidisciplinary team. Some of the faculty felt multiple majors, either engineering or non-engineering, were required. Faculty from other institutions supported the idea that it should focus on the specific background and skill-sets that the team brought to the table. One attendee mentioned, "I would say you don't necessarily want to limit things to just saying just the wording of your discipline, right? You want to look at what there is behind that as well." Another faculty member said that you could still be multidisciplinary within a single degree major, "because imagine maybe the mechanical support from a mechanical design support, mechanical manufacturing support, maybe for mechanical people and different specializations." The faculty ultimately came to a self-directed conclusion that multidisciplinary can lie on a "continuum," which ranges from all in one major with different skill-sets, to various engineering majors, to inclusion of non- 
engineering backgrounds. However, attendees emphasized that institutional availability and programming may limit the extent to which an EPICS team can be multidisciplinary on the continuum. One faculty member actually expressed that if their program could not be classified as multidisciplinary, the department chair would rather the students just take a traditional capstone rather than participate in the EPICS classes; for them, multidisciplinary was essential for program support. On the other hand, faculty from some of the international universities highlighted that their institution had only engineering students, and that student majors are often based off of many societal factors (examination scores, family income, etc.) rather than purely student interest, so it is common for students to develop expertise outside of their major that better aligns with their interests. Additionally, some faculty expressed that while they saw the value of multidisciplinary teams, it was not a priority in their program yet since the communityrelationship and human-centered design experience were more important emphases when starting a program.

The faculty SWOT expanded on many of the themes discussed in the focus group. Strengths identified mimicked the prior themes of skill development and program-level factors such as community partner relationships and interactions. One other identified strength not mentioned in previous conversations but mentioned across multiple groups included access to university resources. Weaknesses of the EPICS program to support multidisciplinary teams were mostly identified as challenges with team dynamics, including one discipline dominating the team, and skills learned such as communication challenges between disciplines or the lack of ability to integrate technical expertise development in order to keep the curriculum broad. Another weakness identified was the focus on engineering in the program name, Engineering Projects in Community Service; some majors were hesitant to join because they were not engineers.

Opportunities tended to focus on program-level factors and the expansion of skill development. Program level factors emphasized by the SWOT analysis included an emphasis on institutionalization (and thus inclusions of more non-engineers) and increasing impact through more community engagement. Skill development opportunities included a larger emphasis on entrepreneurship skills and providing more diverse methods of skill development. Threats were almost exclusively topics of program-level factors, including concerns with community partner interactions and collaborations with other groups within the institution. Multiple groups mentioned challenges such as "losing a community partner" and challenges agreeing with a community partner. Three of the four SWOTs develops focused on the challenge of collaborations within their institutions, including a lack of "buy-in from all departments" and that "academic institutions are hesitant/don't want to change." The faculty SWOT did not show elements of the main themes of motivation for participation or respect for other disciplines.

\subsection{Results of institutional program survey}

Figure 1 shows the data from the institutional program survey regarding the three most important EPICS differentiators for their active EPICS programs. Real projects was the most-often selected choice with $89 \%$ of schools selecting, followed by multi-semester projects $(67 \%)$ and multidisciplinary experience (44\%). Topics written in the "other" category included humancentered design, community partners, and offering skills as students need them. 


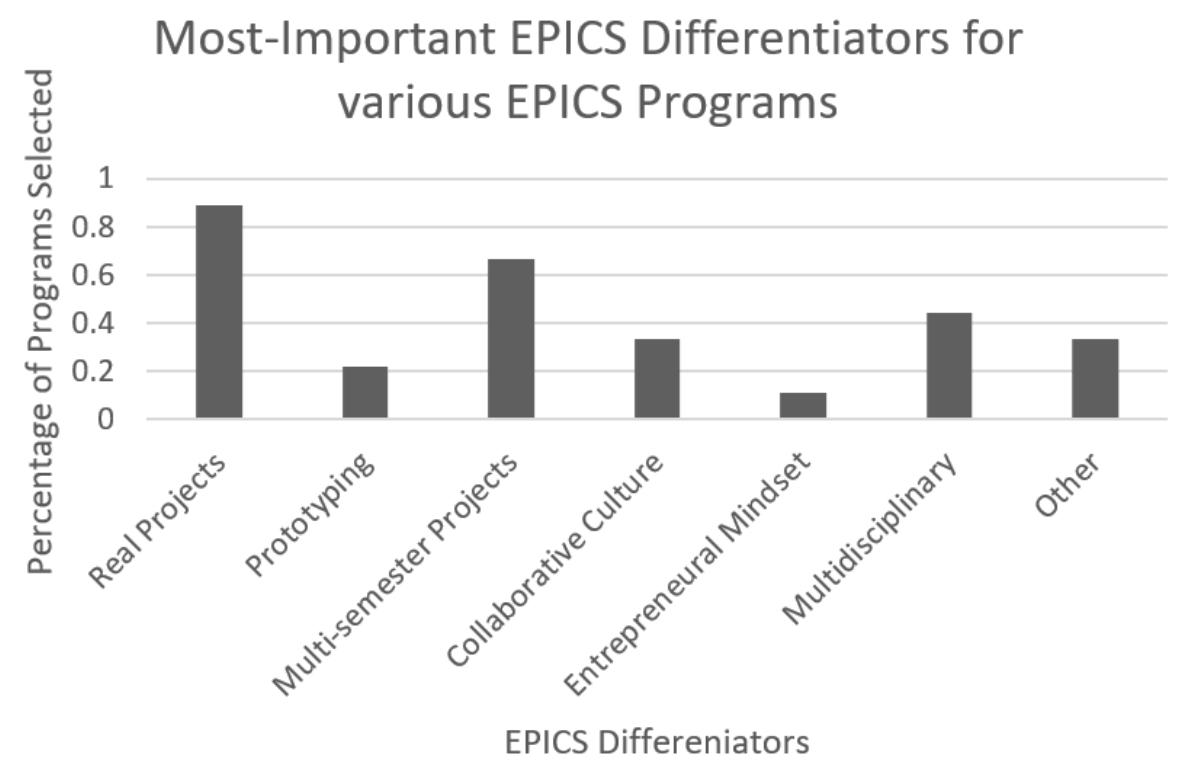

Figure 1: Top 3 EPICS differentiators for various programs with current EPICS programs.

Of the 9 institutions, all of them mentioned residing within some general engineering unit depending upon the organization of their college or university; none were held within a singlediscipline engineering unit such as an electrical engineering department. Program implementations of EPICS also varied: multiple universities integrated EPICS into their first year engineering program, many offered it as an elective, and others have integrated it into required courses. Program sizes ranges from 100 undergraduate students across the entire academic year to 1000 undergraduate students; no institutions currently offered EPICS to their graduate students.

The majority of most EPICS students come from students studying a single engineering major, with often small $(<10 \%)$ percentages of students with double majors or minors inside or outside of engineering. The percentage of non-engineering students varied drastically based on program, with four programs reporting no non-engineering students, two programs with small $(<5 \%)$, two programs with moderate $(<30 \%)$, and one program with a majority of EPICS students as nonengineering students $(75 \%)$.

Program sizes often also vary with respect to the number of teams within an EPICS program. Across an academic year, programs reported supporting between 17 and 85 teams. The majority of programs suggest that at least $50 \%$ of their teams have at least 3 or more types of engineering majors. Three institutions said $100 \%$ of their teams had a non-engineering student on the team, which is often supported through program partnerships within an institution including partnering with global business programs or non-engineering academic majors (i.e., interior design).

One aspect of the EPICS program is inclusion of the course in a student's academic degree requirements. While six of the nine programs allow EPICS to count towards an engineering students' academic degree or elective requirements, only two programs are able to provide similar credit for non-engineering students. Four of the programs mentioned that non- 
engineering students cannot get any degree-credit from their participation in the program. This was sometimes due to the EPICS program being embedded in core engineering courses that would not count towards other degrees (i.e., first year engineering or junior engineering technical requirements). Those programs that are able to offer degree credit for EPICS often mentioned the inclusion of EPICS to fulfill minor requirements or general lower-division humanities requirements.

\section{Discussion}

The six themes identified in this student surveys were a starting point to understand how students viewed multidisciplinary teams in EPICS. Hackman proposed three themes that can represent team success: when a team completes its goals, when the team works together to develop social relations for positive team dynamics, and when individuals feel rewarded for being a member of the team due to social support, new skills, or organizational rewards [14]. The first of these most closely correlates to the code of project success that students emphasized by repeatedly mentioning community partner satisfaction and creating deliverables. The second of these is integrated into the code element of team dynamics, including student comments addressing internal team motivation. The third element can be seen by aligning with the themes of respect for other disciplines and individual skills learned. Social support should come from all majors regardless of background, requiring an acceptance of what each individual member brings to the team. The other two codes (motivation for participation and program-level factors) could be seen as support directly feeding into the other codes and Hackman's themes for team success. While it is possible that students would view their teams as successful without the multidisciplinary aspect, six out of seven students mentioned needing support from those with different backgrounds to be successful in their project.

While all students identified multidisciplinary team members as valuable to an EPICS project, it is clear that it was not one of the main advantages of the EPICS program since it was not mentioned in any of the interviews until brought up by the interviewer. However, once it was brought up, the students tended to identify the positive impacts of multidisciplinary teams, with all seven individuals mentioning the advantages that inclusion of students of other backgrounds to their team. A study by Sharma et al. highlighted that some students perceive multidisciplinary teams to be a barrier to learning rather than an enhancement due to challenges communicating across disciplines [24]. While a few students mentioned some challenges of having students of different backgrounds, most viewed it as a learning opportunity by which to capitalize on others' disciplinary expertise and build communication and leadership skills across backgrounds.

The faculty focus groups highlighted many of the same benefits of incorporating multidisciplinary teams that the students identified, including improved communication skills, an increase in broadening student's perspectives of various disciplines and respecting other ideas, as well as the importance of working with a community partner outside of their area of study for the human-centered design process. However, the faculty group and program-level survey identified many challenges with supporting multidisciplinary teams from a program level. Both the faculty focus group and the program survey indicated that faculty often have many goals for students in the EPICS program, and multidisciplinary teams is not a primary goal. Other program-level objectives include giving students real-world engineering experience, working with community 
partners, and ensuring students walk away with a variety of skill-sets including leadership, communication, and program management, in addition to broadened engineering expertise. Not surprisingly, the program structure within a university can drastic change what support and impact the EPICS program can have across engineering and non-engineering backgrounds. While both students and faculty agree that the inclusion of non-engineering students is an asset to the team, very few programs have successfully integrated EPICS into degree-level coursework requirements for non-engineering majors.

The results of this study should be considered limited-in-scope in a number of ways. The number of participants across all data collection types was relatively small and therefore is only representative of a sampling of EPICS students, faculty, and program-level perspectives on multidisciplinary teams. All student views came from student teams working on food-system solutions in the field of sustainability; while this approach potentially limits differences in student experiences based on project background, it also may result in data that has innate bias towards multidisciplinary teams due to the broad context of the problems tasked to students. Additionally, while best practices for qualitative coding were followed including inter-rater reliability and multiple codebook iterations, results should be considered preliminary and further exploration with students from a larger number of institutions and teams should be considered to validate the results.

\section{Conclusions}

While the main goal of the EPICS program is not to focus on multidisciplinary teams, it is an important differentiator not often seen in other curricular opportunities for students. Students, faculty, and program-directors all agreed that EPICS teams benefit from being multidisciplinary. However, it was also discovered that the term multidisciplinary should be viewed broadly and be recognized as being on a continuum: teams can be successful with a variety of expertise within a single academic major, with engineering-only students, and by including non-engineering students. The level to which a team will be multidisciplinary will often depend on program-level factors and institutional support, including the availability of students of varied backgrounds and the ability to provide academic degree-credit for all participants in the EPICS program. Programs should support students as they learn how to work in multidisciplinary teams by focusing on communication and leadership opportunities with students of different backgrounds, and highlight the transfer of these skills to both industry and academic careers. Additionally, programs are encouraged to work within their institution to build partnerships and relationships with other departments or programs to provide all students the opportunity to work on real world problems for a community organization with a team of diverse backgrounds. 


\section{References}

[1] W. C. Oakes, E. J. Coyle, and L. H. Jamieson, "EPICS: A Model of Service-Learning in an Engineering Curriculum," in Proceedings of 2000 American Society for Engineering Education (ASEE) Annual Conference, St. Louis, MO, June 2000.

[2] Accreditation Board for Engineering and Technology, "Criteria for Accrediting Engineering Programs, 2019 - 2020," [Online]. Available: https://www.abet.org/accreditation/accreditationcriteria/criteria-for-accrediting-engineering-programs-2019-2020/. [Accessed: Jan. 31, 2019].

[3] Accreditation Board for Engineering and Technology, "Criteria for Accrediting Engineering Programs, 2017 - 2018," [Online]. Available: https://www.abet.org/accreditation/accreditationcriteria/criteria-for-accrediting-engineering-programs-2017-2018/. [Accessed: Jan. 31, 2019].

[4] M. A. Collura, B. Aliane, S. Daniels, and J. Nocito-Gobel, "Development of a multidisciplinary engineering foundation spiral," in Proceedings of 2004 American Society for Engineering Education (ASEE) Annual Conference, Salt Lake City, UT, 2004.

[5] L. Shuman, M. Besterfield-Sacre and J. McGourty, "The ABET "Professional Skills" - Can They Be Taught? Can They Be Assessed?", Journal of Engineering Education, vol. 94, no. 1, pp. 41-55, 2005. Available: 10.1002/j.2168-9830.2005.tb00828.x.

[6] M. Borrego and L. K. Newswander, "Definitions of Interdisciplinary Research: Toward Graduate-Level Interdisciplinary Learning Outcomes," Rev. High. Educ., vol. 34, no. 1, pp. 6184, 2010.

[7] K. E. Miller, C. B. Zoltowski, P. M. Buzzanell, D. Torres, D. Corple, and M. Kenny Feister, "Exploring Team Social Responsibility in Multidisciplinary Design Teams" in Proceedings of 2018 American Society for Engineering Education (ASEE) Annual Conference, Salt Lake City, UT, June 2018. https://peer.asee.org/30496

[8] N. Hotaling, B. Fasse, L. Bost, C. Hermann, and C. Forest, "A Quantitative Analysis of the Effects of a Multidisciplinary Engineering Capstone Design Course," Journal of Engineering Education., vol. 101 no. 4, pp. 630-656, 2012.

[9] G. Bucks, W. Oakes, C. Zoltowski, F. DeRego, and S. Mah, "Facilitating Multidisciplinary Teams In A Service Learning Environment," in Proceedings of 2007 American Society for Engineering Education (ASEE) Annual Conference, Honolulu, HI, June 2007. https://peer.asee.org/2551

[10] Frontiers in Education Conference, "Final Program," [online] Available: http://fie2018.org/pages/final-program [accessed Feb 2, 2019].

[11] L. Hong and S. E. Page, "Groups of Diverse Problem Solvers Can Outperform Groups of High-Ability Problem Solvers," in Proceedings of the 2004 National Academy of Sciences of the 
United State of America, vol. 101 iss. 46, pp. 16385-16389, 2004.

https://doi.org/10.1073/pnas.0403723101

[12] A. T. Cummings, J. Huff, W. C. Oakes, and C. B. Zoltowski, "An Assessment Approach to Project-Based Service Learning" in Proceedings of 2013 American Society for Engineering Education (ASEE) Annual Conference and Exposition, Atlanta, GA, June, 2013. https://peer.asee.org/19165

[13] J. L. Schultz, J. R. Wilson, and K. C. Hess, "Team-based Classroom Pedagogy Reframed: The Student Perspective," American Journal of Business Education, vol 3, iss. 7, pp. 17-24, 2013.

[14] J. R. Hackman, "The design of work teams," Handbook of organizational behavior, Englewood Cliffs, NJ: Prentice-Hall, 1987, pp. 315-342,.

[15] D. Levi, Group Dynamics for Teams. Thousand Oaks, CA: Sage Publications, Inc., 2001.

[16] S. Burian, W. Johnson, F. Montague, A. Holt, J. Nielson, and R. David, "Multidiscipline team teaching approach to enhance project-based learning of sustainable design," in Proceedings of 2008 American Society for Engineering Education (ASEE) Annual Conference and Exposition, Pittsburgh, PA, June, 2008.

[17] R. Rabb, J. Hitt, J., and R. Floersheim, "Implementation Of A Complex Multidisciplinary Capstone Project For Stimulating Undergraduate Student Development" in Proceedings of 2010 American Society for Engineering Education (ASEE) Annual Conference and Exposition, Louisville, KY, June, 2010. https://peer.asee.org/15939

[18] R. L. Miller and B. Olds, "A Model Curriculum for a Capstone Course in Multidisciplinary Engineering Design,” Journal of Engineering Education, vol. 83. Iss. 4, pp. 311-316, 1994. 10.1002/j.2168-9830.1994.tb00124.x.

[19] Dedoose Version 8.1.8, "Web Application for Managing, Analyzing, and Presenting Qualitative and Mixed Method Research Data," Los Angeles, CA: SocioCultural Research Consultants, LLC, 2019. [Available at: www.dedoose.com]

[20] V. Braun and V. Clarke, "Using Thematic Analysis in Psychology," Qualitative Research in Psychology, vol. 3, iss. 2, pp. 77-101, 2006.

[21] J. Creswell, "Analyzing and Validating Data," 30 Essential Skills for the Qualitative Researcher, pp. 151-203, 2016.

[22] D. V. Cicchetti, "Guidelines, Criteria, and Rules of Thumb for Evaluating Normed and Standardized Assessment Instruments in Psychology," Psychological Assessment, vol. 6, iss. 4, pp. 284-290, 1994. 
[23] J. L. Fleiss, "Measuring nominal scale agreement among many raters," Psychological Bulletin, vol. 76 iss. 5, pp.378-382, 1971.

[24] B. Sharma, B. Steward, S. K. Ong, and F. E. Miguez, "Evaluation of teaching approach and student learning in a multidisciplinary sustainable engineering course," Journal of Cleaner Production, vol. 142, part 4, pp. 4032-4040, 2017. https://doi.org/10.1016/j.jclepro.2016.10.046 